\title{
Measuring individual inbreeding in the age of genomics: marker-based measures are better than pedigrees
}

\author{
M Kardos ${ }^{1,2}$, G Luikart ${ }^{3}$ and FW Allendorf ${ }^{1}$ \\ Inbreeding (mating between relatives) can dramatically reduce the fitness of offspring by causing parts of the genome to be \\ identical by descent. Thus, measuring individual inbreeding is crucial for ecology, evolution and conservation biology. We used \\ computer simulations to test whether the realized proportion of the genome that is identical by descent $\left(I B D_{\mathrm{G}}\right)$ is predicted \\ better by the pedigree inbreeding coefficient $\left(F_{\mathrm{P}}\right)$ or by genomic (marker-based) measures of inbreeding. Genomic estimators of \\ $I B D_{\mathrm{G}}$ included the increase in individual homozygosity relative to mean Hardy-Weinberg expected homozygosity $\left(F_{\mathrm{H}}\right)$, and two \\ measures $\left(F_{\mathrm{ROH}}\right.$ and $\left.F_{\mathrm{E}}\right)$ that use mapped genetic markers to estimate $I B D_{\mathrm{G}}$. $I B D_{\mathrm{G}}$ was more strongly correlated with $F_{\mathrm{H}}, F_{\mathrm{E}}$ and \\ $F_{\mathrm{ROH}}$ than with $F_{\mathrm{P}}$ across a broad range of simulated scenarios when thousands of SNPs were used. For example, $I B D_{\mathrm{G}}$ was more \\ strongly correlated with $F_{\mathrm{ROH}}, F_{\mathrm{H}}$ and $F_{\mathrm{E}}$ (estimated with $\geqslant 10000 \mathrm{SNPs}$ ) than with $F_{\mathrm{P}}$ (estimated with 20 generations of \\ complete pedigree) in populations with a recent reduction in the effective populations size (from $N_{\mathrm{e}}=500$ to $N_{\mathrm{e}}=75$ ). \\ $F_{\mathrm{ROH}}, F_{\mathrm{H}}$ and $F_{\mathrm{E}}$ generally explained $>90 \%$ of the variance in $I B D_{\mathrm{G}}$ (among individuals) when $35 \mathrm{~K}$ or more SNPs were used. \\ $F_{\mathrm{P}}$ explained $<80 \%$ of the variation in $I B D_{\mathrm{G}}$ on average in all simulated scenarios, even when pedigrees included 20 \\ generations. Our results demonstrate that $I B D_{\mathrm{G}}$ can be more precisely estimated with large numbers of genetic markers than with \\ pedigrees. We encourage researchers to adopt genomic marker-based measures of $I B D_{\mathrm{G}}$ as thousands of loci can now be \\ genotyped in any species.
}

Heredity (2015) 115, 63-72; doi:10.1038/hdy.2015.17; published online 18 March 2015

\section{INTRODUCTION}

Biologists have long recognized that inbred individuals (those whose parents are closely related) often have lower fitness than the offspring of unrelated parents (Darwin, 1868; Ives and Whitlock, 2002). The cumulative effects of inbreeding on individual fitness can reduce the population growth rate and the probability of persistence (O'Grady et al., 2006; Kenney et al., 2014). Effects of inbreeding on individual fitness and population dynamics make measuring individual inbreeding a crucial part of many studies in ecology, evolution and conservation biology.

Inbred individuals have lower genome-wide heterozygosity because a fraction of loci are 'identical by descent' (IBD). A locus is identical by descent if it carries two gene copies that both originated from a single copy in a common ancestor of the parents. All measures of individual inbreeding seek to predict the proportion of the genome that is identical by descent $\left(I B D_{\mathrm{G}}\right)$. The classical measure of individual inbreeding is the pedigree inbreeding coefficient $F_{\mathrm{P}}$ (Wright, 1922; Keller and Waller, 2002). $F_{\mathrm{P}}$ predicts the $I B D_{\mathrm{G}}$ due to the known common ancestors of parents and assumes that the pedigree founders are unrelated and non-inbred. $F_{\mathrm{P}}$ has often been considered as the best measure of individual inbreeding (Pemberton, 2004,2008). However, $F_{\mathrm{P}}$ may be an imprecise measure of $I B D_{\mathrm{G}}$ because $I B D_{\mathrm{G}}$ can vary substantially among individuals with the same pedigree (for example, siblings, Figure 1 and Supplementary Figure S1) (Franklin, 1977; Hill and Weir, 2011; Forstmeier et al., 2012). The standard deviation of $I B D_{\mathrm{G}}$ among individuals with the same pedigree is highest in species with few chromosomes and short genetic maps (Franklin, 1977; Stam, 1980; Hill and Weir, 2011; Figure 1 and Supplementary Figure S1). Furthermore, $F_{\mathrm{P}}$ cannot account for inbreeding caused by distant ancestors not included in a pedigree.

The increasing availability of large-scale molecular genetic data might make it possible to more precisely estimate $I B D_{\mathrm{G}}$ with genetic markers than with pedigrees (Keller et al., 2011; Hoffman et al., 2014). For example, $I B D_{\mathrm{G}}$ can be estimated as the increase in individual homozygosity relative to Hardy-Weinberg expected homozygosity $\left(F_{\mathrm{H}}\right)$ (Purcell et al., 2007). Other measures of $I B D_{\mathrm{G}}$ use mapped genetic markers to identify IBD chromosome segments (referred to here as IBD tracts), which are characterized by long contiguous runs of homozygosity $(\mathrm{ROH})$ at mapped genetic markers (Chapman and Thompson, 2003; McQuillan et al., 2008). The $F_{\mathrm{ROH}}$ statistic estimates $I B D_{\mathrm{G}}$ as the proportion of the genome within inferred $\mathrm{ROH}$ that satisfy user-defined criteria (for example, number of SNPs, physical length and SNP density) (Purcell et al., 2007; McQuillan et al., 2012). Leutenegger et al. (2003) introduced a maximum likelihood estimator $\left(F_{\mathrm{E}}\right)$ that uses mapped genetic markers and a hidden Markov model to identify putative IBD tracts and to estimate $I B D_{\mathrm{G}}$.

A thorough evaluation of the relative performance of pedigreeversus marker-based measures of $I B D_{\mathrm{G}}$ in small populations would advance our understanding of how individual inbreeding should be measured in natural populations of conservation concern as we enter an era when thousands of loci can be genotyped for any organism.

${ }^{1}$ Division of Biological Sciences, University of Montana, Missoula, MT, USA; ${ }^{2}$ Department of Evolutionary Biology, Evolutionary Biology Centre (EBC), Uppsala University, Uppsala, Sweden and ${ }^{3}$ Flathead Lake Biological Station, Division of Biological Sciences, University of Montana, Polson, MT, USA 

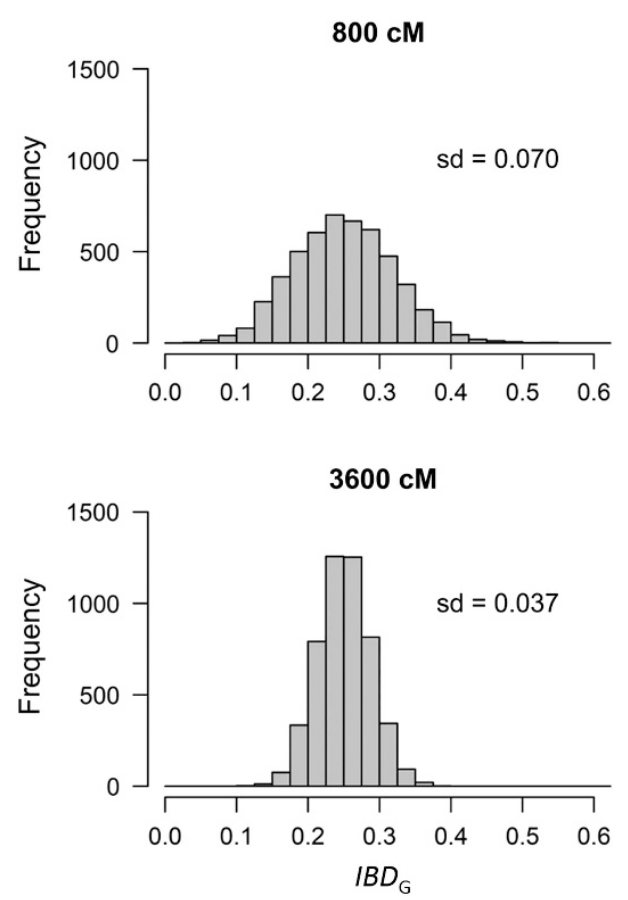

Figure 1 The distribution of $I B D_{\mathrm{G}}$ among 5000 simulated offspring of full siblings with 20 chromosomes of equal length, and $800 \mathrm{~cm}$ (upper panel) or 3600 cm (lower panel) genomes.

Using simulations, Keller et al. (2011) found that the number of homozygous rare alleles within an individual was more strongly correlated with marker-based measures of inbreeding than with $F_{\mathrm{P}}$. However, they simulated large populations with $220 \mathrm{~cm}$ genomes and only 2 chromosomes. Thus, their results likely underestimate the precision of $F_{\mathrm{P}}$ as a measure of $I B D_{\mathrm{G}}$ in humans and other species with much longer genetic maps and more chromosomes (Figure 1 and Supplementary Figure S1; Franklin, 1977).

Our objective was to test whether $I B D_{\mathrm{G}}$ is predicted better by pedigrees or by genetic markers in small populations. Specifically, we evaluated the precision and bias of $F_{\mathrm{P}}, F_{\mathrm{ROH}}, F_{\mathrm{E}}$ and $F_{\mathrm{H}}$ in small populations that are of the greatest concern for conservation with genomic characteristics typical of mammals. Our simulations accounted for the depth of the pedigree used to estimate $F_{\mathrm{P}}$, the number of markers used to estimate $F_{\mathrm{ROH}}, F_{\mathrm{E}}$ and $F_{\mathrm{H}}$, the genetic map length of the genome and demographic history. We emphasize comparisons of the performance of $F_{\mathrm{P}}$ versus the marker-based measures of $I B D_{\mathrm{G}}$.

\section{MATERIALS AND METHODS}

\section{Computer simulation model}

We used a stochastic, individual-based simulation program (Kardos et al., 2014) for R version 3.0.1 ( $\mathrm{R}$ Core Team, 2013). We simulated a sexually reproducing, random mating, non-selfing species with nonoverlapping generations. We simulated hermaphrodites to avoid temporal fluctuations in the effective populations size $\left(N_{\mathrm{e}}\right)$ due to stochastic variation in the sex ratio. We simulated recombination and kept track of the ancestral origin of each chromosome segment, which was known to the base pair. We simulated genomic characteristics typical of mammals, including $3 \mathrm{~Gb}$ genomes with 20 chromosomes of equal size, and mutation with a base change probability of $1.2 \times 10^{-8}$ per base pair per generation. We simulated only 20 chromosomes because chromosome number does not strongly affect the standard deviation of $I B D_{\mathrm{G}}$ among individuals with the same pedigree $\left.\sigma\left(I B D_{\mathrm{G}}\right)\right)$ when genetic map lengths are in the range we simulated (Supplementary Figure S1). We used two different recombination rates to evaluate the effect of genetic map length on the precision of $F_{\mathrm{P}}, F_{\mathrm{ROH}}, F_{\mathrm{E}}$ and $F_{\mathrm{H}}$. We simulated genomes with $1.2 \mathrm{~cm} / \mathrm{Mb}$ (that is, an average of approximately 0.012 recombinations per $\mathrm{Mb}$ per meiosis) which is similar to humans (Dumont and Payseur, 2008) and resulted in a genetic map length of $3600 \mathrm{~cm}$. We also simulated genomes with $0.27 \mathrm{~cm} / \mathrm{Mb}$ which is typical of the lower end of the distribution of recombination rates among mammals (Dumont and Payseur, 2008) and resulted in a genetic map length of $800 \mathrm{~cm}$.

Our model of recombination follows the simulations of Chapman and Thompson (2003) and is based on Fisher's theory of junctions (Fisher, 1965). We assume no interference, that the number of crossovers along a chromosome is Poisson distributed, and that the recombination probability is constant across the genome and among individuals.

Population founders were unrelated and non-inbred. Thus, $I B D_{\mathrm{G}}$ for an individual is relative to the population founders. Founders for each simulated population were drawn from a hypothetical source population in Hardy-Weinberg equilibrium. Allele frequencies at $400 \mathrm{~K}(K=1000)$ SNPs in the source populations were assigned so that the mean expected heterozygosity $\left(H_{\mathrm{e}}\right)$ was approximately 0.3 at the end of the simulations before SNP filtering steps (see below). To account for the effects of SNP heterozygosity (Miller et al., 2014) on our results, we repeated simulations of populations with $3600 \mathrm{~cm}$ genomes with founder allele frequencies assigned so that $H_{\mathrm{e}}$ was only approximately 0.2 , and analyzed these data without filtering based on minor allele frequency (MAF) (beyond removing fixed loci). SNP positions were determined using a random number generator so that each nucleotide position in the genome was equally likely to be polymorphic in the source population. Individual founder genotypes were assigned by randomly choosing two alleles at each locus based on their frequencies in the source population (see Kardos et al., 2014 for further details on the assignment of founder genotypes). Simulation output included $I B D_{\mathrm{G}}$ and genotypes at $300-350 \mathrm{~K}$ diallelic SNPs, which were left after removing loci with $\mathrm{MAF}<0.05$ for each individual in the final generation. SNPs with $\mathrm{MAF}<0.05$ were removed to maximize the information content of the genotypes. Statistical analyses were performed only on individuals from the last generation to hold the number of pedigree generations used to estimate $F_{\mathrm{P}}$ constant among individuals within each analysis, and to hold the sample sizes for statistical tests constant across all simulations. An R script for the simulations is available in Dryad (see Data Accessibility).

\section{Defining IBD $_{\mathrm{G}}$}

We defined $I B D_{\mathrm{G}}$ as the realized proportion of the physical genome in IBD tracts. $I B D_{\mathrm{G}}$ was measured without error because the exact boundaries of IBD tracts were known to the base pair. Thus, $I B D_{\mathrm{G}}$ is a parameter representing the true individual genomic inbreeding. The $I B D_{\mathrm{G}}$ was calculated from the simulated genomic data once for each individual. The mean and variance of $I B D_{\mathrm{G}}$ for each simulated scenario are shown in Supplementary Table S2.

\section{Simulated demographic scenarios}

We simulated two demographic scenarios to account for differences in the variance of $I B D_{\mathrm{G}}$, and to mimic populations where researchers are often interested in inbreeding and its effects on fitness. First, we simulated partially isolated populations with a constant local $N_{\mathrm{e}}$ of 75 for 150 generations, receiving occasional immigrants $(m=0.013$, one 
immigrant per generation on average). This scenario mimics small populations on habitat islands with occasional immigration from a large source population. Immigrants were unrelated to each other and to residents. Immigrants were drawn from the same hypothetical source population as the founders and thus had genotypes drawn from the same allele frequency distribution as the founders. Second, we simulated closed populations that were large (constant $N_{\mathrm{e}}=500$ ) for 90 generations, and experienced a reduction in size to $N_{\mathrm{e}}=75$ for 10 additional generations. We ran 20 replicate simulations for each scenario.

We wanted to test whether our results were substantially different when all inbreeding was due to recent ancestors. Therefore, we reran the simulations as described above, except now running the simulations for only 20 generations (not 100-150 generations as above). Here, all inbreeding is due to recent (within 20 generations) ancestors, and $F_{\mathrm{P}}$ estimated with a 20 generation pedigree accounts for all common ancestors of parents. For these simulations, partially isolated populations had a constant local $N_{\mathrm{e}}$ of 75 for 20 generations, and received one immigrant per generation on average $(m=0.013)$. Populations with recently reduced $N_{\mathrm{e}}$ were again closed $(m=0)$ and had $N_{\mathrm{e}}=500$ for the first 10 generations, then $N_{\mathrm{e}}=75$ for the last 10 generations.

\section{Linkage disequilibrium pruning}

Before estimating $F_{\mathrm{ROH}}, F_{\mathrm{E}}$ and $F_{\mathrm{H}}$, we used the-indep-pairwise function in PLINK version 1.07 (Purcell et al., 2007) to reduce linkage disequilibrium among the 300-350 K SNPs remaining after MAFbased filtering. We conducted 'LD pruning' because $F_{\mathrm{E}}$ assumes all markers are in linkage equilibrium (Polašek et al., 2010). Additionally, $F_{\mathrm{ROH}}$ is typically estimated after LD pruning to avoid detecting fixed or nearly fixed $\mathrm{ROH}$ arising due to ancient population processes (for example, selective sweeps) (for example, McQuillan et al., 2012). For LD pruning, we used a sliding window of 25 SNPs, a step size of 10 SNPs and we removed one SNP from each pair in a window above the $\mathrm{LD}$ threshold of $r^{2}=0.5$. Our results were not substantively affected by increasing the size of the sliding window for LD pruning to 50 SNPs, or by using a more stringent LD pruning threshold $\left(r^{2}=0.25\right)$ (data not shown).

We conducted statistical analyses on 500 SNPs to $100 \mathrm{~K}$ SNPs randomly sampled from those remaining after MAF- and LD-based filtering. We used only up to $100 \mathrm{~K}$ SNPs because preliminary simulations showed that using more loci did not substantively affect our results (data not shown). Mean $H_{\mathrm{e}}$ after filtering based on MAF and LD was 0.31 in partially isolated populations with $3600 \mathrm{~cm}$ genomes, and 0.33 on average in partially isolated populations with $800 \mathrm{~cm}$ genomes. $H_{\mathrm{e}}$ was 0.35 on average in populations with recently reduced $N_{\mathrm{e}}$. These levels of SNP heterozygosity are similar to large SNP chip data sets from mammals and birds (for example, Deatwyler et al., 2010; Kawakami et al., 2014). In our simulations of populations with low heterozygosity SNPs $\left(H_{\mathrm{e}} \approx 0.2\right)$, the mean realized $H_{\mathrm{e}}$ after removing fixed loci and $\mathrm{LD}$ pruning was 0.21 in populations with recently reduced $N_{\mathrm{e}}$ and 0.19 in partially isolated populations.

\section{Estimators of $\mathrm{IBD}_{\mathrm{G}}$}

We used three marker-based measures of $I B D_{\mathrm{G}}$. We used the-homozyg function in PLINK to estimate $F_{\mathrm{ROH}}$ using $5-100 \mathrm{~K}$ SNPs. PLINK uses a sliding window approach and user defined criteria to identify $\mathrm{ROH}$, which are inferred to be putative IBD tracts. $F_{\mathrm{ROH}}$ is estimated as the proportion of the genome in $\mathrm{ROH}$, and ranges from zero to one. Thus, $F_{\mathrm{ROH}}$ is directly proportional to $I B D_{\mathrm{G}}$. We used only $\geqslant 5 \mathrm{~K}$ SNPs to estimate $F_{\mathrm{ROH}}$ because we believed that SNP density would be too low to reliably detect $\mathrm{ROH}$ when fewer loci are used, regardless of the parameter settings used in PLINK. For example, fewer than two SNPs are expected to occur in an 8-Mb IBD tract on average when only 500 randomly distributed SNPs are used in a 3-Gb genome. We adjusted the required number of SNPs and SNP density (maximum $\mathrm{Kb}$ per SNP on average within an $\mathrm{ROH}$ ) per inferred $\mathrm{ROH}$ based on the number of loci used (Supplementary Table S1). We allowed a single heterozygous position in any inferred $\mathrm{ROH}$ to account for the occasional mutation occurring within otherwise IBD chromosome segments. Initial testing showed that adjusting PLINK parameters according to the number of SNPs and LD pruning substantially improved the performance of $F_{\mathrm{ROH}}$ when $\leqslant 25 \mathrm{~K}$ SNPs were used. Consistent with our definition of $I B D_{\mathrm{G}}$ above, we estimated $F_{\mathrm{ROH}}$ as the sum of the lengths of all detected $\mathrm{ROH}$ detected by PLINK divided by the physical genome size $(3 \mathrm{~Gb})$.

The second measure of $I B D_{\mathrm{G}}$ we used $\left(F_{\mathrm{H}}\right)$ measures the excess in the observed number of homozygous genotypes within an individual relative to the mean number of homozygous genotypes expected under random mating:

$$
F_{H_{i}}=\frac{O\left(\text { Hom }_{i}\right)-E(H o m)}{m-E(H o m)},
$$

where $\mathrm{O}\left(\mathrm{Hom}_{\mathrm{i}}\right)$ is the observed number of homozygous loci for the $i$ th individual, andE(Hom) is the Hardy-Weinberg expected mean number of homozygous genotypes across $m$ loci (Keller et al., 2011). The average $O(H o m)$ is expected to equal $E(H o m)$ on average in random mating populations. $F_{\mathrm{H}}$ can thus take negative values (like some other marker-based measures of inbreeding or relatedness; Wang, 2014), and will be centered near zero in random mating populations when allele frequencies are taken from the current sample of individuals. $F_{\mathrm{H}}$ should thus be considered as a proxy rather than a direct estimator of $I B D_{\mathrm{G}}$, which is a proportion with range $0-1$. We estimated $F_{\mathrm{H}}$ using the-het function in PLINK.

The third marker-based estimator $\left(F_{\mathrm{E}}\right)$ is implemented in the program FEstim and uses maximum likelihood and a hidden Markov model to determine the values of $I B D_{\mathrm{G}}$ that maximize the likelihood of the observed genotypes at mapped loci (Leutenegger et al., 2003). $F_{\mathrm{E}}$ has a theoretical range of $0-1$ and is thus directly proportional to $I B D_{\mathrm{G}}$. Starting values for both $F_{\mathrm{E}}$ and the IBD rate of change parameter were 0.1. Default FEstim settings were used for other parameters. FEstim did not consistently run to completion on data from partially isolated populations when more than $10 \mathrm{~K}$ SNPs were used, even when using more stringent LD pruning settings in PLINK (LD threshold of $r^{2}=0.25$, and a sliding window size of 50 SNPs). This is likely due to higher LD remaining among closely linked SNPs in the partially isolated populations than in populations with recently reduced $N_{\mathrm{e}}$ after LD pruning (Supplementary Figure S2). Using more stringent LD pruning thresholds (for example, $r^{2}=0.1$ ) left too few SNPs to usefully compare $F_{\mathrm{E}}$ and the other $I B D_{\mathrm{G}}$ estimators. Thus, we present results from $F_{\mathrm{E}}$ in partially isolated populations for $10 \mathrm{~K}$ and fewer SNPs only. Allele frequencies used to estimate $F_{\mathrm{H}}$ and $F_{\mathrm{E}}$ were from the 75 individuals in the last generation of each simulation. We used the kinship2 package in $\mathrm{R}$ to estimate $F_{\mathrm{P}}$ for each individual using 3-20 generations of pedigree.

\section{Comparing the performance of $F_{\mathrm{P}}, F_{\mathrm{H}}, F_{\mathrm{E}}$ and $F_{\mathrm{ROH}}$}

We used the proportion of variance in $I B D_{\mathrm{G}}$ explained by $F_{\mathrm{ROH}}, F_{\mathrm{E}}$, $F_{\mathrm{H}}$ and $F_{\mathrm{P}}\left(r^{2}\right)$ from linear regression models to evaluate the precision of $F_{\mathrm{ROH}}, F_{\mathrm{E}}, F_{\mathrm{H}}$ and $F_{\mathrm{P}}(N=$ all 75 individuals from the last simulated generation). We conducted separate regressions of $F_{\mathrm{ROH}}, F_{\mathrm{H}}, F_{\mathrm{E}}$ and $F_{\mathrm{P}}$ versus $I B D_{\mathrm{G}}$ for individuals in the final generation of each simulated population. We used two-tailed $t$-tests to determine whether 
the mean $r^{2}$ from regressions with $I B D_{\mathrm{G}}$ (among 20 replicate simulations) was statistically significantly different for $F_{\mathrm{ROH}}, F_{\mathrm{H}}, F_{\mathrm{E}}$ and $F_{\mathrm{P}}$. We compared all possible combinations of pedigree depth (for $F_{\mathrm{P}}$ ) and number of SNP used (for $F_{\mathrm{H}}, F_{\mathrm{E}}$, and $F_{\mathrm{ROH}}$ ) when testing if the mean $r^{2}$ with $I B D_{\mathrm{G}}$ was statistically significantly different for $F_{\mathrm{P}}, F_{\mathrm{H}}, F_{\mathrm{E}}$ and $F_{\mathrm{ROH}}$. We also used $t$-tests to determine whether the mean $r^{2}$ from regressions with $I B D_{\mathrm{G}}$ was statistically significantly different for $F_{\mathrm{ROH}}$, $F_{\mathrm{E}}, F_{\mathrm{H}}$ and $F_{\mathrm{P}}$ when the genetic map length was $800 \mathrm{~cm}$ instead of $3600 \mathrm{~cm}$, and when simulations were run for 20 generations instead of 100-150 generations.

We measured bias of the $I B D_{\mathrm{G}}$ estimators as the mean amount by which $F_{\mathrm{P}}, F_{\mathrm{H}}, F_{\mathrm{E}}$ and $F_{\mathrm{ROH}}$ over- or under-estimated $I B D_{\mathrm{G}}$ (that is, the mean of the values of $F_{\mathrm{P}} F_{\mathrm{H}}, F_{\mathrm{E}}$ or $F_{\mathrm{ROH}}$ minus $\left.I B D_{\mathrm{G}}\right)$. Note that $F_{\mathrm{H}}$ is expected to underestimate $I B D_{\mathrm{G}}$ when allele frequencies are taken from the current population and mating occurs at random. Thus, we expect $F_{\mathrm{H}}$ to underestimate $I B D_{\mathrm{G}}$ in our simulated random mating populations. We also wanted to measure bias of the estimated differences in $I B D_{\mathrm{G}}$ among individuals. For this we estimated the ratio of the standard deviation of each of the estimators $\left(F_{\mathrm{P}}, F_{\mathrm{H}}, F_{\mathrm{E}}\right.$ and $\left.F_{\mathrm{ROH}}\right)$ to the standard deviation of $I B D_{\mathrm{G}}$ to measure bias of the estimated differences in $I B D_{\mathrm{G}}$ among individuals. A ratio of the standard deviation of an estimator to the standard deviation of $I B D_{\mathrm{G}}$ near 1 indicates that the estimated differences in $I B D_{\mathrm{G}}$ among individuals are unbiased. Ratios of below or above 1 indicate that estimated differences in $I B D_{\mathrm{G}}$ among individuals are substantially under- or overestimated.

\section{RESULTS}

We first present results on the precision and bias of $F_{\mathrm{P}}, F_{\mathrm{H}}, F_{\mathrm{E}}$ and $F_{\mathrm{ROH}}$ in simulations of $3600 \mathrm{~cm}$ genomes where inbreeding was due to both recent and distant ancestors (that is, simulations ran for 100-150 generations). We then summarize how the results change when inbreeding is due only to recent ancestors (that is, simulations ran for only 20 generations), when genetic map length is shortened to $800 \mathrm{~cm}$, and finally when SNP $H_{\mathrm{e}} \approx 0.2$ (instead of $H_{\mathrm{e}} \approx 0.3$ ).

\section{Precision of $I B D_{G}$ estimators}

$F_{\mathrm{P}}$ had low precision in both demographic scenarios (Figure 2). The mean $r^{2}$ from regressions of $F_{\mathrm{P}}$ versus $I B D_{\mathrm{G}}$ across 20 simulation repetitions $\left(\bar{r}_{F_{P}-I B D_{G}}^{2}\right)$ was 0.72 in partially isolated populations and 0.71 in populations with recently reduced $N_{\mathrm{e}}$ when pedigrees included 20 generations. Using more than five pedigree generations had little effect on $\bar{r}_{F_{P}-I B D_{G}}^{2}$ in populations with recently reduced $N_{\mathrm{e}}$. However, $\bar{r}_{F_{P}-I B D_{G}}^{2}$ increased when deeper pedigrees were used in partially isolated populations.

$F_{\mathrm{ROH}}$ and $F_{\mathrm{H}}$ had high precision in both demographic scenarios when large number of SNPs were used. The mean $r^{2}$ from regressions with $I B D_{\mathrm{G}}$ was statistically significantly higher for $F_{\mathrm{H}}\left(\bar{r}_{F_{H}-I B D_{G}}^{2}\right)$ than for $F_{\mathrm{E}}\left(\bar{r}_{F_{E}-I B D_{G}}^{2}\right)$ or $F_{\mathrm{ROH}}\left(\bar{r}_{F_{R O H}-I B D_{G}}^{2}\right)$ in partially isolated populations for all numbers of loci used $(P<0.05$, Figure 2$) . \bar{r}_{F_{H}-I B D_{G}}^{2}$ was $>0.9$ when $10 \mathrm{~K}$ or more SNPs were used in both demographic scenarios. $\bar{r}_{F_{R O H}-I B D_{G}}^{2}$ was $>0.9$ when $100 \mathrm{~K}$ SNPs were used in partially isolated populations, and when $\geqslant 15 \mathrm{~K}$ SNPs were used in populations with recently reduced $N_{\mathrm{e}} \cdot \bar{r}_{F_{E}-I B D_{G}}^{2}$ was always statistically significantly

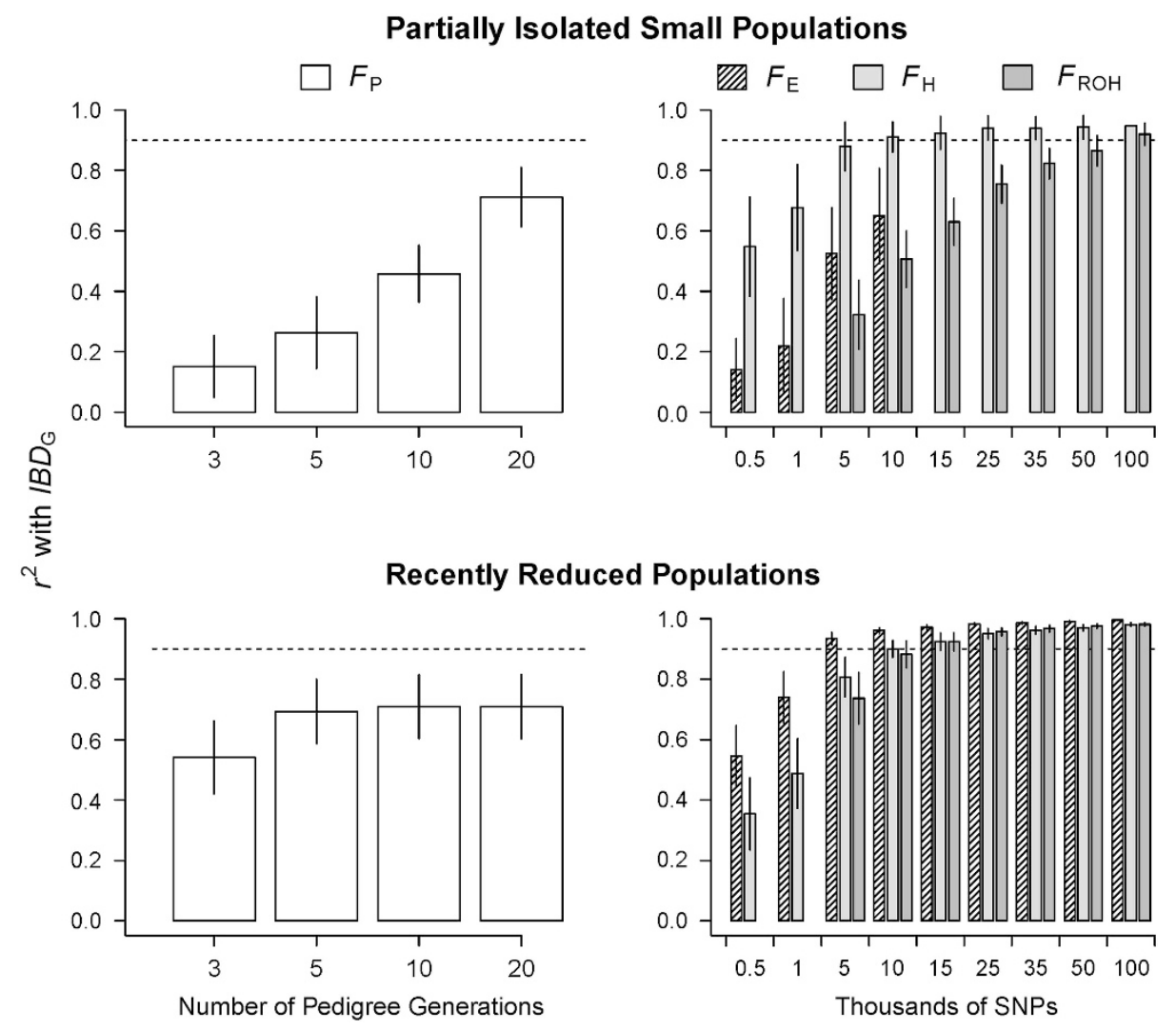

Figure 2 Barplots of the mean $r^{2}\left( \pm 1\right.$ s.d. among 20 simulated populations) from regressions of $F_{\mathrm{P}}, F_{\mathrm{E}}, F_{\mathrm{H}}$ and $F_{\mathrm{ROH}}$ versus $I B D_{\mathrm{G}}$. The data shown are from 20 partially isolated populations (top row) and also 20 populations with recently reduced $N_{\mathrm{e}}$ (bottom row) and $3600 \mathrm{~cm}$ genomes. Dashed lines at $r^{2}=0.9$ are to aid comparison of $r^{2}$ for $F_{\mathrm{P}}, F_{\mathrm{H}}, F_{\mathrm{E}}$ and $F_{\mathrm{ROH}}$. 
higher than $\bar{r}_{F_{H}-I B D_{G}}^{2}$ and $\bar{r}_{F_{R O H}-I B D_{G}}^{2}$ in populations with recently reduced $N_{\mathrm{e}}(P<0.001)$.

$F_{\mathrm{ROH}}, F_{\mathrm{E}}$ and $F_{\mathrm{H}}$ were always more precise than $F_{\mathrm{P}}$ when large numbers of SNPs were used. For example, $\bar{r}_{F_{H}-I B D_{G}}^{2}$ measured with $\geqslant 5 \mathrm{~K}$ SNPs and $\bar{r}_{F_{R O H}-I B D_{G}}^{2}$ measured with $\geqslant 35 \mathrm{~K}$ SNPs were both statistically significantly higher than $\bar{r}_{F_{p}-I B D_{G}}^{2}$ based on 20 generation pedigrees in partially isolated populations $(P<0.001$; Figure 2$)$. The mean $r^{2}$ from regressions with $I B D_{\mathrm{G}}$ was statistically significantly higher for $F_{\mathrm{ROH}}, F_{\mathrm{E}}$ and $F_{\mathrm{H}}$ estimated with $\geqslant 10 \mathrm{~K}$ SNPs than for $F_{\mathrm{P}}$ estimated with 20 generations in populations with recently reduced $N_{\mathrm{e}}$ $(P<0.001)$

\section{Bias of $\mathrm{IBD}_{\mathrm{G}}$ estimators}

$F_{\mathrm{P}}$ consistently underestimated $I B D_{\mathrm{G}}$ in both simulated demographic scenarios (Figure 3). For example, $F_{\mathrm{P}}$ underestimated $I B D_{\mathrm{G}}$ by 0.19 with 3 generation pedigrees, and by 0.1 with 20 generation pedigrees on average in partially isolated populations. $F_{\mathrm{P}}$ was less downwardly biased in populations with recently reduced $N_{\mathrm{e}}$ where $F_{\mathrm{P}}$ underestimated $I B D_{\mathrm{G}}$ by 0.13 and 0.07 on average when pedigrees included 3 and 20 generations, respectively.

Marker-based estimators were all substantially downwardly biased when few SNPs were used, and this downward bias was largest in partially isolated populations (Figure 3 ). $F_{\mathrm{E}}$ and $F_{\mathrm{ROH}}$ became less downwardly biased when larger numbers of SNPs were used. For example, $F_{\mathrm{E}}$ underestimated $I B D_{\mathrm{G}}$ by 0.11 when 500 SNPs were used, and by only 0.02 when $100 \mathrm{~K}$ SNPs were used in populations with recently reduced $N_{\mathrm{e}}$. $F_{\mathrm{ROH}}$ underestimated $I B D_{\mathrm{G}}$ by 0.14 when $5 \mathrm{~K}$ SNPs were used, and by 0.05 on average when $100 \mathrm{~K}$ SNPs were used in populations with recently reduced $N_{\mathrm{e}} . F_{\mathrm{H}}$ was the most downwardly biased of all $(P<0.0001)$ and the number of SNPs had no effect on bias. $F_{\mathrm{H}}$ underestimated $I B D_{\mathrm{G}}$ by 0.21 on average in partially isolated populations and by 0.16 in populations with recently reduced $N_{\mathrm{e}}$.

Both $F_{\mathrm{E}}$ and $F_{\mathrm{ROH}}$ were less downwardly biased than $F_{\mathrm{P}}$ in both demographic scenarios when large numbers of SNPs were used (Figure 3). $F_{\mathrm{ROH}}$ estimated with $10 \mathrm{~K}$ and $50 \mathrm{~K}$ SNPs was less downwardly biased than $F_{\mathrm{P}}$ estimated with 3 and 20 generations, respectively, in populations with recently reduced $N_{\mathrm{e}}(P<0.0001)$. $F_{\mathrm{E}}$ estimated with 500 and $10 \mathrm{~K}$ SNPs was less downwardly biased than $F_{\mathrm{P}}$ estimated with 3 and 20 generations, respectively, in populations with recently reduced $N_{\mathrm{e}}(P<0.0001) . F_{\mathrm{H}}$ had larger downward bias than $F_{\mathrm{P}}$ in all cases (Figure 3 ). The precision and bias of $F_{\mathrm{ROH}}, F_{\mathrm{E}}, F_{\mathrm{H}}$ and $F_{\mathrm{P}}$ relative to $I B D_{\mathrm{G}}$ in a representative population is shown in Figure 4.

Bias in estimated differences in $\mathrm{IBD}_{\mathrm{G}}$ among individuals

The mean ratio of the standard deviation of $F_{\mathrm{P}}$ to the standard deviation of $I B D_{\mathrm{G}}\left(\overline{\sigma_{F_{P}} / \sigma_{I B D_{G}}}\right)$ across 20 simulation repetitions was $<1$ in all scenarios (Figure 5), $\overline{\sigma_{F_{p}} / \sigma_{I B D_{G}}}$ was 0.61 in partially isolated populations and 0.86 in populations with recently reduced $N_{\mathrm{e}}$ when pedigrees included 20 generations. Using pedigrees of more than five generations did not substantively affect $\overline{\sigma_{F_{P}} / \sigma_{I B D_{G}}}$ in populations with recently reduced $N_{\mathrm{e}}$.

Partially Isolated Small Populations
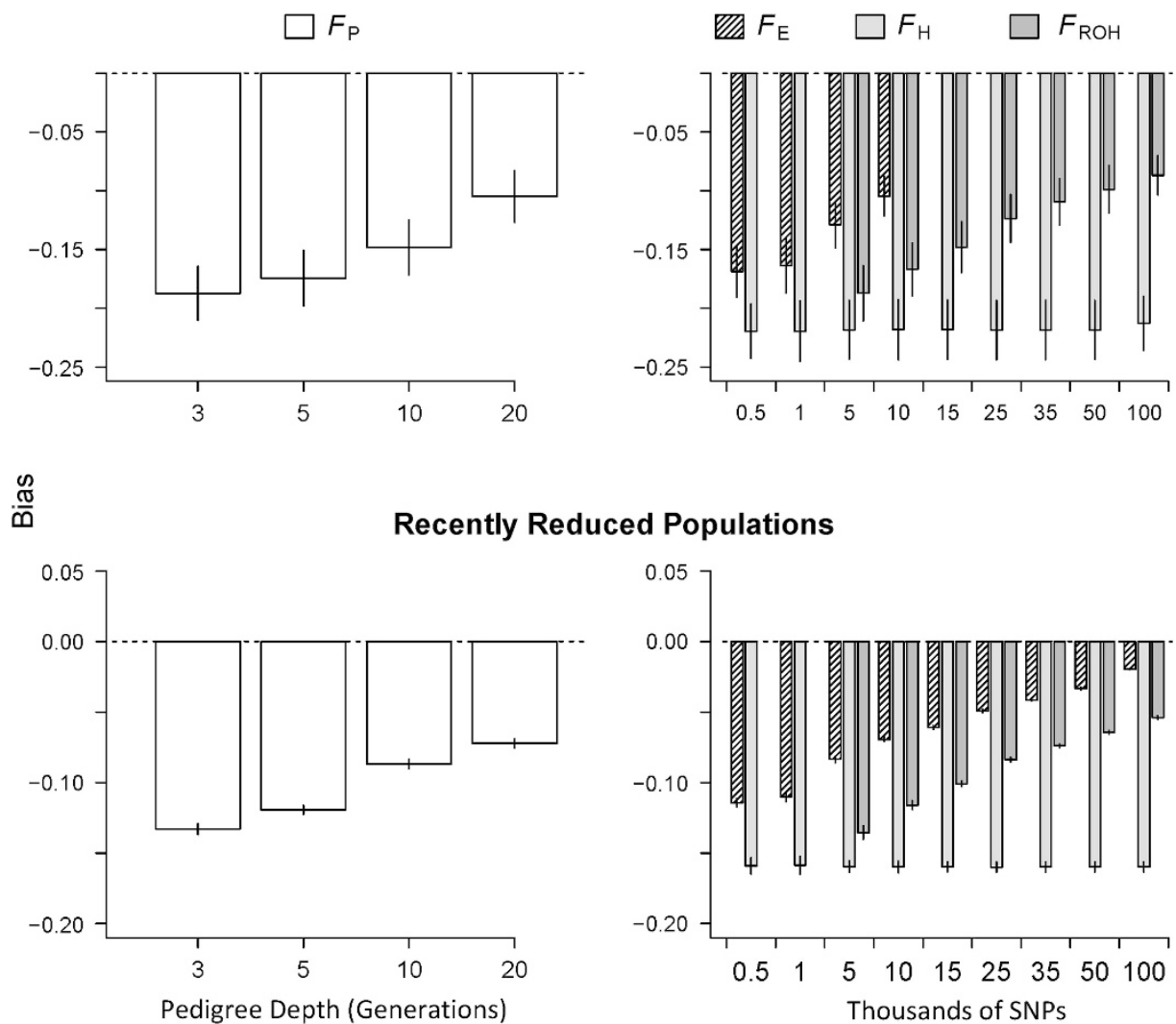

Figure 3 Barplots of the mean bias of $F_{\mathrm{P}}, F_{\mathrm{H}}, F_{\mathrm{E}}$ and $F_{\mathrm{ROH}}\left( \pm 1\right.$ s.d. among 20 simulated populations). Bias was measured as the mean error $\left(F_{\mathrm{P}}, F_{\mathrm{H}}, F_{\mathrm{E}}\right.$ or $F_{\mathrm{ROH}}$ minus $\left(B D_{\mathrm{G}}\right)$ across all individuals in each simulated population. The data shown are from 20 partially isolated populations (top row) and 20 populations with recently reduced $N_{\mathrm{e}}$ (bottom row) and $3600 \mathrm{~cm}$ genomes. 
a

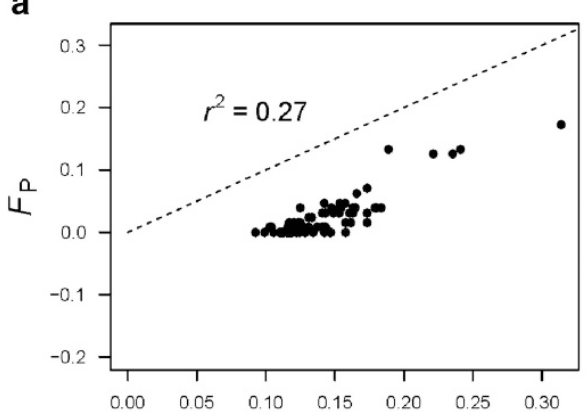

C

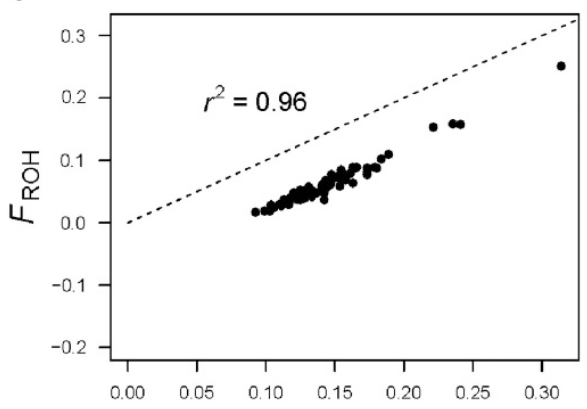

b

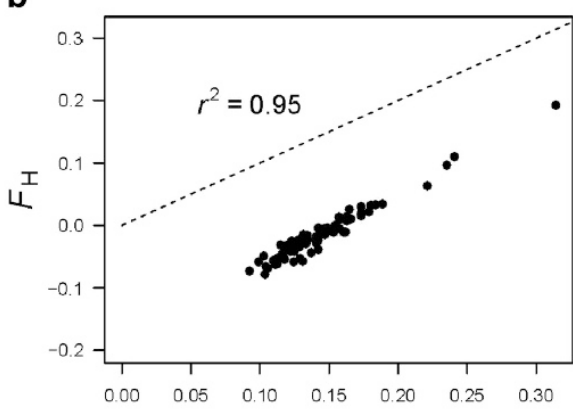

d

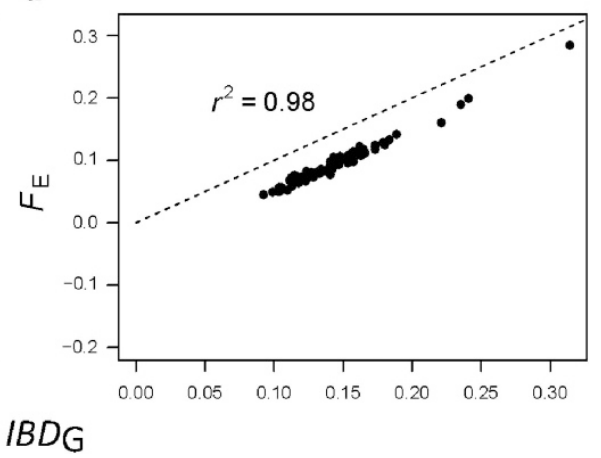

Figure $4 F_{\mathrm{P}}\left(\right.$ a), $F_{\mathrm{H}}(\mathbf{b}), F_{\mathrm{ROH}}(\mathrm{c})$ and $F_{\mathrm{E}}$ (d) versus $I B D_{\mathrm{G}}$ in a population with recently reduced $N_{\mathrm{e}}$ and $3600 \mathrm{~cm}$ genomes. $F_{\mathrm{P}}$ was estimated with five generations. $F_{\mathrm{H}}, F_{\mathrm{E}}$ and $F_{\mathrm{ROH}}$ were estimated with $25 \mathrm{~K}$ SNPs. The dashed lines have intercept of zero and slope of one. Points below the lines represent underestimates of $I B D_{\mathrm{G}}$.

\section{Partially Isolated Small Populations}

$F_{\mathrm{P}}$

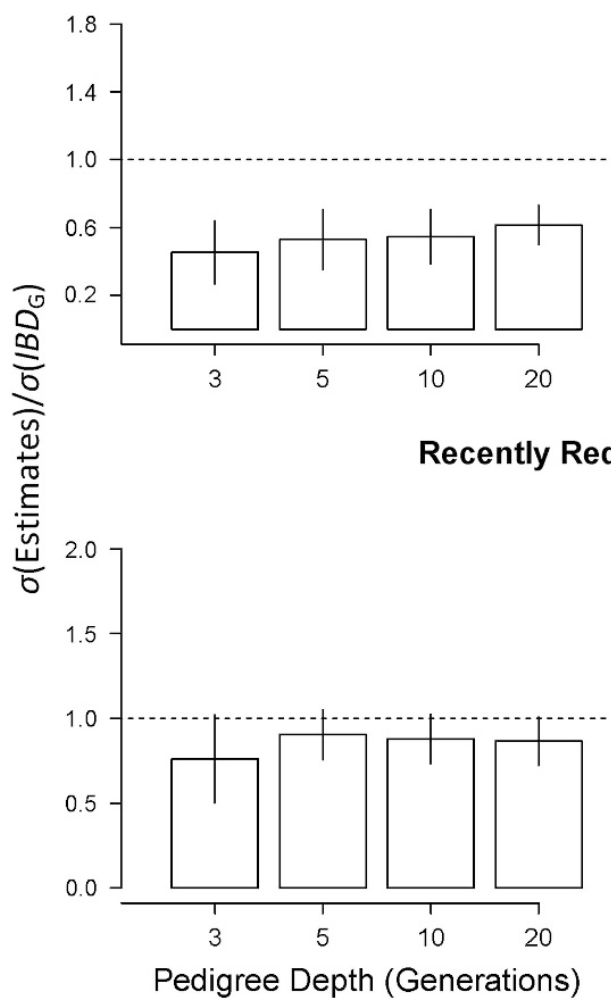

Pedigree Depth (Generations)
$\mathbb{Z} F_{\mathrm{E}} \quad \square F_{\mathrm{H}}$

$F_{\mathrm{ROH}}$

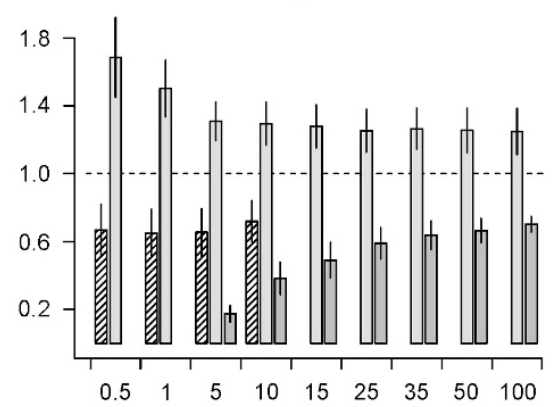

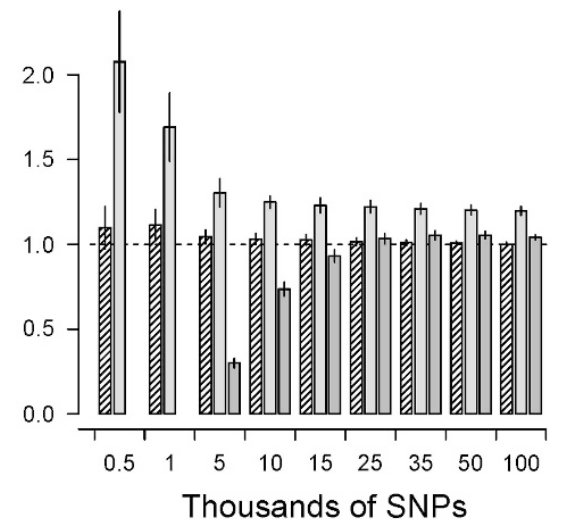

Figure 5 Ratios of the standard deviation of $F_{\mathrm{P}}, F_{\mathrm{E}}, F_{\mathrm{H}}$ and $F_{\mathrm{ROH}}$ to the standard deviation of $I B D_{\mathrm{G}}$. The data shown are from partially isolated populations (top row) and populations with recently reduced $N_{\mathrm{e}}$ (bottom row) and $3600 \mathrm{~cm}$ genomes. The dashed lines represent a ratio of 1 . Bars extending above and below the line represent over- and underestimates, respectively, of the difference in $I B D_{\mathrm{G}}$ among individuals. 
$F_{\mathrm{H}}$ always overestimated the differences in $I B D_{\mathrm{G}}$ among individuals, particularly when relatively few SNPs were used (Figure 5). For example, the mean ratio of the standard deviation of $F_{\mathrm{H}}$ to the standard deviation of $I B D_{\mathrm{G}}\left(\overline{\sigma_{F_{H}} / \sigma_{I B D_{G}}}\right)$ across 20 simulation repetitions was approximately 1.7 when $5 \mathrm{~K}$ SNPs were used, and 1.25 when $15 \mathrm{~K}$ SNPs were used in partially isolated populations.

The mean ratio of the standard deviation of $F_{\mathrm{ROH}}$ to the standard deviation of $I B D_{\mathrm{G}}\left(\overline{\sigma_{F_{R O H}} / \sigma_{I B D_{G}}}\right)$ was consistently below 1 in partially isolated populations (Figure 5), $\overline{\sigma_{F_{R O H}} / \sigma_{I B D_{G}}}$ was 0.70 when $100 \mathrm{~K}$ SNPs were used in partially isolated populations. However, in populations with recently reduced $N_{\mathrm{e}}, \overline{\sigma_{F_{R O H}} / \sigma_{I B D_{G}}}$ was approximately 1.05 on average when $\geqslant 25 \mathrm{~K}$ SNPs were used (Figure 5). The mean ratio of the standard deviation of $F_{\mathrm{E}}$ to the standard deviation of $I B D_{\mathrm{G}}$ $\left(\overline{\sigma_{F_{E}} / \sigma_{I B D_{G}}}\right)$ was 1.1 when 500 SNPs were used, and 1.0 when $100 \mathrm{~K}$ SNPs were used in populations with recently reduced $N_{\mathrm{e}}$ (Figure 5). However, $\overline{\sigma_{F_{E}} / \sigma_{I B D_{G}}}$ was approximately 0.72 with $10 \mathrm{~K}$ SNPs in partially isolated populations.

$\overline{\sigma_{F_{E}} / \sigma_{I B D_{G}}}$ and $\overline{\sigma_{F_{R O H}} / \sigma_{I B D_{G}}}$ were always $>\overline{\sigma_{F_{P}} / \sigma_{I B D_{G}}}$ when large numbers of SNPs were used (Figure 5). For example, $\overline{\sigma_{F_{E}} / \sigma_{I B D_{G}}}$ was statistically significantly higher than $\overline{\sigma_{F_{P}} / \sigma_{I B D_{G}}}$ estimated with 20 generations when $10 \mathrm{~K}$ SNPs were used in partially isolated populations $(P=0.009)$ and when $\geqslant 500$ SNPs were used in populations with recently reduced $N_{\mathrm{e}}(P<0.0001) . \quad \overline{\sigma_{F_{R O H}} / \sigma_{I B D_{G}}}$ was statistically significantly higher than $\overline{\sigma_{F_{P}} / \sigma_{I B D_{G}}}$ estimated with 20 generations when $100 \mathrm{~K}$ SNPs were used in partially isolated populations $(P=0.003)$, and when $\geqslant 15 \mathrm{~K}$ SNPs were used in populations with recently reduced $N_{\mathrm{e}}(P \leqslant 0.025)$.

In the following sections, we summarize how altering simulation parameters (genetic map length, number of simulated generations and SNP heterozygosity) caused results to differ substantially from those presented above. Detailed results from these scenarios are provided in Supplementary Figures S3-S14.

\section{Effects of the genetic map length on precision and bias}

$\bar{r}_{F_{p}-I B D_{G}}^{2}$ was always statistically significantly lower in populations with $800 \mathrm{~cm}$ genomes than in populations with $3600 \mathrm{~cm}$ genomes $(P \leqslant 0.005)$, except when pedigrees included only $3-5$ generations in partially isolated populations (Figure 2 and Supplementary Figure S3). For example, $\bar{r}_{F_{P}-I B D_{G}}^{2}$ based on three pedigree generations was 0.54 when the genetic map was $3600 \mathrm{~cm}$, and 0.36 when the genetic map was $800 \mathrm{~cm}$ in populations with recently reduced $N_{\mathrm{e}}$.

$F_{\mathrm{E}}$ and $F_{\mathrm{ROH}}$ always had statistically significantly higher precision in partially isolated populations with $800 \mathrm{~cm}$ genomes than with $3600 \mathrm{~cm}$ genomes $(P<0.002)$, with the exception of when $F_{\mathrm{ROH}}$ was estimated with $100 \mathrm{~K}$ SNPs (Figure 2 and Supplementary Figure S3). For example $\bar{r}_{F_{E}-I B D_{G}}^{2}$ and $\bar{r}_{F_{R O H}-I B D_{G}}^{2}$ were 0.83 and 0.88 , respectively, when $10 \mathrm{~K}$ SNPs were used in partially isolated populations with $800 \mathrm{~cm}$ genomes. However, $\bar{r}_{F_{E}-I B D_{G}}^{2}$ and $\vec{r}_{F_{R O H}-I B D_{G}}^{2}$ were 0.65 and 0.5 , respectively, when $10 \mathrm{~K}$ SNPs in partially isolated populations with $3600 \mathrm{~cm}$ genomes. $F_{\mathrm{ROH}}$ and $F_{\mathrm{E}}$ were often statistically significantly less downwardly biased when genomes were $800 \mathrm{~cm}$ instead of $3600 \mathrm{~cm}$, but these differences in bias were always small $(\leqslant 0.06)$ (Figure 3 and Supplementary Figure S4).

$\overline{\sigma_{F_{P}} / \sigma_{I B D_{G}}}$ based on pedigrees including five or more generations was always statistically significantly lower when genomes were $800 \mathrm{~cm}$ than when genomes were $3600 \mathrm{~cm}$ in populations with recently reduced $N_{\mathrm{e}}(P \leqslant 0.001$; Figure 5 and Supplementary Figure S5). For example, $\overline{\sigma_{F_{P}} / \sigma_{I B D_{G}}}$ based on pedigrees including five generations was 0.90 with $3600 \mathrm{~cm}$ genomes, and 0.75 with $800 \mathrm{~cm}$ genomes in populations with recently reduced $N_{\mathrm{e}} \cdot \overline{\sigma_{F_{R O H}} / \sigma_{I B D_{G}}}$ was 0.2 units higher (closer to 1 ) on average in partially isolated populations with
$800 \mathrm{~cm}$ genomes than in partially populations with $3600 \mathrm{~cm}$ genomes $\left(P<0.0001\right.$, Figure 5 and Supplementary Figure S5). $\overline{\sigma_{F_{E}} / \sigma_{I B D_{G}}}$ was 0.16 units higher (closer to 1 ) on average with $800 \mathrm{~cm}$ genomes than with $3600 \mathrm{~cm}$ genomes in partially isolated populations $(P<0.002)$.

\section{Performance of $\mathrm{IBD}_{\mathrm{G}}$ estimators when inbreeding is due only to recent ancestors}

$F_{\mathrm{P}}$ was imprecise when all inbreeding was due to recent ancestors (that is, in simulations run for only 20 generations). For example, $\bar{r}_{F_{p}-I B D_{G}}^{2}$ estimated with 20 generation pedigrees was 0.78 in partially isolated populations with $3600 \mathrm{~cm}$ genomes (Supplementary Figure S6) and 0.53 in partially isolated populations with $800 \mathrm{~cm}$ genomes (Supplementary Figure S9).

The precision of the marker-based measures of $I B D_{\mathrm{G}}$ in 20 generation simulations was generally very similar to the results described above for simulations that ran for 100-150 generations (Supplementary Figures S6 and S9). However, $\bar{r}_{F_{E}-I B D_{G}}^{2}$ was considerably higher in partially isolated populations when the simulations ran for only 20 generations instead of 150 generations (Figure 2 and Supplementary Figure S6).

$F_{\mathrm{ROH}}, F_{\mathrm{E}}, F_{\mathrm{H}}$ and $F_{\mathrm{P}}$ had lower bias in simulations that ran for only 20 generations (Supplementary Figures S7 and S10) than in simulations that ran for 100-150 generations (Figure 3 and Supplementary Figure $S 4$ ). In particular, $F_{\mathrm{P}}$ estimated with pedigrees including all 20 generations was unbiased, as expected when pedigree founders are unrelated and non-inbred.

The standard deviations of $F_{\mathrm{ROH}}, F_{\mathrm{E}}$ and $F_{\mathrm{P}}$ more closely matched the standard deviation of $I B D_{\mathrm{G}}$ in simulations of partially isolated populations that ran for only 20 generations instead of 150 generations (Figure 5 and Supplementary Figure S8). For example, $\overline{\sigma_{F_{P}} / \sigma_{I B D_{G}}}$ was always $<0.65$ in partially isolated populations with $3600 \mathrm{~cm}$ genomes simulated for 150 generations. However, $\overline{\sigma_{F_{P}} / \sigma_{I B D_{G}}}$ was $>0.8$ when $F_{\mathrm{P}}$ was estimated with $\geqslant 5$ generations of pedigree in partially isolated populations simulated for only 20 generations. $\overline{\sigma_{F_{E}} / \sigma_{I B D_{G}}}$ and $\overline{\sigma_{F R O H} / \sigma_{I B D_{G}}}$ were always $<0.75$ in partially isolated populations run for 150 generations. However, in partially isolated populations simulated for only 20 generations, $\overline{\sigma_{F_{E}} / \sigma_{I B D_{G}}}$ was $>0.9$ for all numbers of loci used, and $\overline{\sigma_{F R O H} / \sigma_{I B D_{G}}}$ was $>0.9$ when $F_{\mathrm{ROH}}$ was estimated with $100 \mathrm{~K}$ SNPs.

\section{Effects of SNP heterozygosity}

$F_{\mathrm{H}}$ and $F_{\mathrm{ROH}}$ were less precise in simulations with lower heterozygosity $\left(H_{\mathrm{e}} \approx 0.2\right)$ when relatively few loci were used. For example, $\bar{r}_{F_{R O H}-I B D_{G}}^{2}$ was 0.35 with $H_{\mathrm{e}} \approx 0.2$ and 0.74 with $H_{\mathrm{e}} \approx 0.3$ when $5 \mathrm{~K}$ SNPs were used in populations with $3600 \mathrm{~cm}$ genomes and recently reduced $N_{\mathrm{e}}$ (Figure 2 and Supplementary Figure S12). However, the number of SNPs necessary for high precision $\left(r^{2} \geqslant 0.9\right)$ was unaffected by SNP heterozygosity for any of the marker-based measures of $I B D_{\mathrm{G}}$.

\section{DISCUSSION}

Pedigrees have long been the preferred tool to measure individual inbreeding (Pemberton, 2004,2008). However, we found that the realized IBD proportion of the genome $\left(I B D_{\mathrm{G}}\right)$ is better predicted by large numbers of genetic markers than by pedigrees. Our results are consistent with two recent studies that used different approaches to evaluate the precision of marker- versus pedigree-based measures of individual inbreeding when using thousands of SNPs. Hoffman et al. (2014) found a moderately high correlation between heterozygosity estimated at $>13000 \mathrm{SNPs}$ and $F_{\mathrm{P}}\left(r^{2}=0.74\right)$ in oldfield mice (Peromyscus polionotus). However, the predicted $r^{2}$ between heterozygosity and $I B D_{\mathrm{G}}$ (based on estimated identity disequilibrium in their 
oldfield mice) was $\approx 1$; thus, $I B D_{\mathrm{G}}$ was likely more precisely predicted by heterozygosity than by $F_{\mathrm{P}}$ in their study population. Keller et al. (2011) found that the number of homozygous rare alleles within an individual was more strongly correlated with marker-based estimates of inbreeding than with $F_{\mathrm{P}}$ in large simulated populations with short genetic maps $(220 \mathrm{~cm})$ and two chromosomes.

We expanded on previous work (Keller et al., 2011; Hoffman et al., 2014) by evaluating the performance of marker- and pedigree-based measures of $I B D_{\mathrm{G}}$ using simulations where $I B D_{\mathrm{G}}$ was known for each individual without error. Additionally, we accounted for variation in the genetic map length, population demography, heterozygosity of the genetic markers and pedigree depth. Finally, we specifically simulated relatively small populations that are of the greatest concern for conservation. Our results suggest that marker-based measures of $I B D_{\mathrm{G}}$ computed from thousands of loci should be preferred over measures based on $F_{\mathrm{P}}$ in future studies of inbreeding depression in natural populations.

\section{Precision and bias}

$F_{\mathrm{P}}$ had low precision and underestimated the differences in $I B D_{\mathrm{G}}$ among individuals in all simulated scenarios. Additionally, $F_{\mathrm{P}}$ was always downwardly biased except when pedigrees included 20 generations in simulations that ran for only 20 generations and pedigree founders were thus unrelated and non-inbred (Supplementary Figures S7 and S10). Errors in $F_{\mathrm{P}}$ have two major sources. As discussed above, $\sigma\left(I B D_{\mathrm{G}}\right)$ can be high among individuals with the same pedigree (Figure 1; Franklin, 1977). High $\sigma\left(I B D_{\mathrm{G}}\right)$ partially explains why multiple locus heterozygosity is usually only weakly related to $F_{\mathrm{P}}$, and may also explain why heterozygosity-fitness correlations are sometimes observed when $F_{\mathrm{P}}$-fitness correlations are absent (Forstmeier et al., 2012). Linkage and a finite genetic map length are expected to weaken the correlation between $F_{\mathrm{P}}$ and $I B D_{\mathrm{G}}$, but not to cause $F_{\mathrm{P}}$ to be downwardly biased.

The downward bias in $F_{\mathrm{P}}$ can easily be explained by relatedness and inbreeding of pedigree founders. When founders are related, inbreeding due to common ancestors deeper in an individual's ancestry is not accounted for, thus causing $F_{\mathrm{P}}$ to underestimate $I B D_{\mathrm{G}} . F_{\mathrm{P}}$ will also underestimate $I B D_{\mathrm{G}}$ for individuals whose parents have a common ancestor that is an inbred founder. Moving the base population further back in time (that is, including more generations in a pedigree) accounts for a larger proportion of the common ancestors of parents and the inbreeding of those common ancestors, thus decreasing the downward bias of $F_{\mathrm{P}}$ (Figure 3 and Supplementary Figure S15). High variance in founder relatedness may reduce the precision of $F_{\mathrm{P}}$ by causing variation among individuals in the magnitude of the underestimation of $I B D_{\mathrm{G}}$. A focus of future research should be to determine the relative influence of related or inbred pedigree founders versus linkage and recombination on the imprecision of $F_{\mathrm{P}}$.

All of the marker-based measures of $I B D_{\mathrm{G}}$ were highly precise when large number of SNPs were used. Additionally, genetic markers showed lower bias than $F_{\mathrm{P}}$ in estimated differences in $I B D_{\mathrm{G}}$ among individuals. Thousands of SNPs can currently be typed for any organism, for example, with SNP chips (for example, Kawakami et al., 2014), restriction site-associated DNA sequencing (RAD-seq) (Davey et al., 2011), or by whole genome sequencing (Ellegren, 2014). These technologies along with the increasing number of whole genome reference assemblies make it possible to precisely measure $I B D_{\mathrm{G}}$ with molecular markers in any species. Genotyping large numbers of SNPs is still expensive, but this cost will almost always pale in comparison with the expense and difficulty of obtaining deep pedigrees in free-ranging natural populations.
$F_{\mathrm{H}}$ was always very strongly correlated with $I B D_{\mathrm{G}}\left(r^{2}\right.$ near or above 0.9 ) when $10 \mathrm{~K}$ or more SNPs were used (Figure 2 and Supplementary Figure S3). However, as expected, $F_{\mathrm{H}}$ underestimated $I B D_{\mathrm{G}}$ (Figure 3 and Supplementary Figure S4) and the magnitude of this bias was unaffected by the number of SNPs. This bias can be explained by a discrepancy between the assumed and actual base populations used to estimate $F_{\mathrm{H}}$ (Wang, 2014). As mentioned above, $F_{\mathrm{H}}$ can take negative values, and is expected to be centered near zero when allele frequencies are estimated from the current population and mating is random (for example, Figure 4). For $F_{\mathrm{H}}$ to provide unbiased estimates of $I B D_{\mathrm{G}}$, allele frequencies must be derived from a base population where all individuals would be unrelated if mating occurred randomly (Supplementary Figure S16). Allele frequencies usually can only be estimated using the individuals for which inbreeding measurements are desired. Mean pairwise relatedness can be high in populations with small $N_{\mathrm{e}}$ (Wang, 2014), which are often the focus of studies of inbreeding and inbreeding depression. Thus, researchers should expect $F_{\mathrm{H}}$ to substantially underestimate $I B D_{\mathrm{G}}$ in most small natural populations.

$F_{\mathrm{P}}$ consistently underestimated the differences in $I B D_{\mathrm{G}}$ among individuals (Figure 5 and Supplementary Figure S5). This is likely due to pedigrees failing to account for the variation in $I B D_{\mathrm{G}}$ due to distant ancestors, and linkage and recombination. We found that $F_{\mathrm{ROH}}$ and $F_{\mathrm{E}}$ had lower bias than $F_{\mathrm{P}}$ in estimated differences in $I B D_{\mathrm{G}}$ among individuals when large numbers of SNPs were used (for example, Figure 5). Downward bias in estimates of the differences in $I B D_{\mathrm{G}}$ (among individuals) might lead to overestimation of the strength of inbreeding depression (Keller, 1998) because of a greater decrease in fitness per unit increase in $F_{\mathrm{P}}$ than per unit increase in $I B D_{\mathrm{G}}$. This suggests that the magnitude of inbreeding depression (that is, the number of lethal equivalents, Keller and Waller, 2002) might be overestimated in pedigree-based tests for inbreeding depression. However, estimates of the number of lethal equivalents might have lower bias when using $F_{\mathrm{ROH}}$ and $F_{\mathrm{E}}$ with large numbers of SNPs. The statistical performance of marker- versus pedigree-based estimates of the strength of inbreeding depression should be formally evaluated in future research.

\section{How many SNPs are needed?}

The number of markers necessary for precise and low-bias estimates of $I B D_{\mathrm{G}}$ in a particular study system will depend on the genome size, whether inbreeding due to distant ancestors is of interest, marker heterozygosity and proportion of missing genotypes, and the variance of $I B D_{\mathrm{G}}$. The ability to reliably detect IBD tracts depends on marker density (SNPs/cm); thus, the necessary number of markers increases with genome map length. Additionally, higher density markers are necessary to detect short IBD tracts caused by distant ancestors or a very high recombination rate (Supplementary Figure S17). The expected correlation between heterozygosity-based measures of individual inbreeding and $I B D_{\mathrm{G}}$ increases with heterozygosity and the variance of $I B D_{\mathrm{G}}$ (Miller et al., 2014). This was evident in our results where $F_{\mathrm{ROH}}$ and $F_{\mathrm{H}}$ often had higher precision when $H_{\mathrm{e}}$ was high. Thus, more loci may be necessary for marker-based measures of $I B D_{\mathrm{G}}$ to have higher precision than $F_{\mathrm{P}}$ in studies where $H_{\mathrm{e}}$ is low.

The discussion above suggests that there is not a single number of loci that will always achieve high precision and low bias estimates of $I B D_{\mathrm{G}}$. Simulations with genomic and demographic parameters set to match a particular study system can be used to evaluate the number of markers necessary to precisely estimate $I B D_{\mathrm{G}}$. However, we believe the number of SNPs typically available with SNP chips or RAD-seq $\left(\geqslant 10^{4}\right.$ SNPs, Allendorf et al., 2010) should in most cases provide highly 
precise estimates of $I B D_{\mathrm{G}}$ when used with one or more of the markerbased measures of $I B D_{\mathrm{G}}$ included in the present study.

\section{Effects of genetic map length on the performance of IBD $_{G}$ estimators}

We found that the advantages of marker-based measures of $I B D_{\mathrm{G}}$ over $F_{\mathrm{P}}$ are greatest in organisms with short genetic map lengths. The reduced precision of $F_{\mathrm{P}}$ among organisms with shorter map lengths is easily attributed to the higher $\sigma\left(I B D_{\mathrm{G}}\right)$ because of fewer crossovers per meiosis on average (Franklin, 1977). Unlike $F_{\mathrm{P}}, F_{\mathrm{E}}$ and $F_{\mathrm{ROH}}$ were often more strongly correlated with $I B D_{\mathrm{G}}$ in populations with shorter genetic maps (Figure 2 and Supplementary Figure S3). The increased precision of $F_{\mathrm{E}}$ and $F_{\mathrm{ROH}}$ in populations with shorter genetic maps likely occurs because the longer IBD tracts resulting from a lower recombination rate (Supplementary Figure S17) are more easily detected by the hidden Markov model and sliding window algorithms used to estimate $F_{\mathrm{ROH}}$ and $F_{\mathrm{E}}$. These results demonstrate that the marker-based measures of $I B D_{\mathrm{G}}$ are sensitive to the higher variance in $I B D_{\mathrm{G}}$ in organisms with shorter genetic maps. Therefore, markerbased measures of $I B D_{\mathrm{G}}$ should be even more strongly preferred over $F_{\mathrm{P}}$ in organisms with short genetic maps.

We simulated genomes typical of mammals in terms of the physical size $(3 \mathrm{~Gb})$, number of chromosomes and genetic map length (800-3600 cM; Dumont and Payseur, 2008). $F_{\mathrm{P}}$ is will be less precise in species with shorter genetic maps or fewer chromosomes due to higher variance in $I B D_{\mathrm{G}}$ among individuals with the same pedigree (Franklin, 1977; Supplementary Figure S1). Thus, the advantage of marker-based measures of $I B D_{\mathrm{G}}$ over $F_{\mathrm{P}}$ is expected to be greater in species with fewer chromosomes or shorter genetic maps. However, the precision of $F_{\mathrm{P}}$ will increase in species with longer genetic maps or more chromosomes, and more loci will be required to have equivalent or higher precision than $F_{\mathrm{P}}$ in such cases.

\section{Relative value of $F_{\mathrm{ROH}}, F_{\mathrm{E}}$ and $F_{\mathrm{H}}$}

The major advantages of $F_{\mathrm{H}}$ are that it does not require mapped genetic markers and it was highly precise in all simulated scenarios when using as few as $10 \mathrm{~K}$ SNPs (Figure 2 and Supplementary Figure S3). Physical genome maps are currently available for few taxa (Ellegren, 2014). Therefore, our results are encouraging for studies on organisms lacking genome maps and have no choice but to measure individual inbreeding with unmapped loci (for example, Hoffman et al., 2014).

Though $I B D_{\mathrm{G}}$ can be measured precisely with unmapped genetic markers, there are advantages to using mapped markers when they are available. In addition to showing high precision and low bias when large numbers of markers are used, $F_{\mathrm{ROH}}$ can distinguish inbreeding due to recent versus distant ancestors by including only specific ROH length categories in statistical analyses (for example, Kirin et al., 2010). Inbreeding depression could be caused mainly by inbreeding due to recent ancestors when a large fraction of deleterious recessive alleles are purged over many generations. For example, Szpeich et al. (2013) found that very long $\mathrm{ROH}$ (caused mainly by recent ancestors) was more highly enriched for deleterious recessive alleles than short $\mathrm{ROH}$ (caused mainly by distant ancestors) in humans. Therefore, tests for inbreeding depression may often be more powerful when $F_{\mathrm{ROH}}$ is estimated only with very long ROH. However, it could be important to account for inbreeding due to distant ancestors (for example, by including short $\mathrm{ROH}$ in $F_{\mathrm{ROH}}$, or by using $F_{\mathrm{E}}$ or $F_{\mathrm{H}}$ ) when purging is inefficient at removing the genetic load (for example, when inbreeding depression is caused by many deleterious recessive alleles with small effects).
$F_{\mathrm{E}}$ had higher precision and often lower bias than $F_{\mathrm{H}}$ and $F_{\mathrm{ROH}}$ when relatively few markers were used (for example, $\leqslant 10 \mathrm{~K} \mathrm{SNPs}$ ) (Figures 2,3 and 5). Therefore, $F_{\mathrm{E}}$ may often be the most powerful measure of $I B D_{\mathrm{G}}$ in studies employing relatively few mapped SNPs. However, the current implementation of $F_{\mathrm{E}}$ (FEstim) almost always failed to run to completion when $>10 \mathrm{~K}$ SNPs were used in partially isolated populations, presumably due to high $\mathrm{LD}$ remaining among linked SNPs (Supplementary Figure S2). Lowering the $N_{\mathrm{e}}$ of the simulated populations from $N_{\mathrm{e}}=75$ to $N_{\mathrm{e}}=20$, which increases the magnitude of LD (Hill, 1981), resulted in FEstim failing to run to completion in the great majority of simulation repetitions in both demographic scenarios (data not shown). This suggests that studies of populations with very small $N_{\mathrm{e}}$ (where strong LD extends across the entire genome) will often be limited to using small numbers of SNPs with FEstim.

\section{Assumptions and limitations}

Our simulations assumed no crossover interference. However, interference can be strong in some vertebrates (Broman and Weber, 2000). We are not aware that the effects of interference on the spatial and length distributions of IBD tracts have been investigated. Therefore, it is unclear how interference affects the performance of pedigree- and marker-based measures of $I B D_{\mathrm{G}}$.

We also assumed pedigrees and genotypes were error free. However, genotyping (Pompanon et al., 2005) and pedigree errors (Pemberton, 2008) are common. Incorrect heterozygous versus homozygous calls will increase errors in marker-based statistics. Likewise, incorrect or missing parentage assignments will increase errors in $F_{\mathrm{P}}$ (Pemberton, 2008). The effects of genotyping and pedigree errors were beyond the scope of this study. However, plausible rates of genotyping or pedigree errors should be considered in empirical inbreeding studies. Further, we did not simulate inbreeding depression. It is not clear how inbreeding depression, where the most highly inbred individuals are less likely to survive and be sampled in natural populations, might affect the performance of marker- and pedigree-based measures of $I B D_{\mathrm{G}}$. Finally, we simulated genetic markers with $H_{\mathrm{e}}$ in the approximate range of $0.2-0.3$. Using loci with lower heterozygosity is expected to decrease the precision of marker-based estimates of $I B D_{\mathrm{G}}$.

\section{CONCLUSIONS}

Our results show that marker-based measures of $I B D_{\mathrm{G}}$ are substantially more precise and often less biased than $F_{\mathrm{P}}$. This and other recent findings (for example, Hoffman et al., 2014; Keller et al., 2011) are in stark contrast to previous studies that used few genetic markers and did not consider the effects of linkage and recombination on the relative performance of pedigree- versus marker-based measures of inbreeding (for example, Balloux et al., 2004; Slate et al., 2004; Santure et al., 2010). The current explosion of DNA sequence data from nonmodel organisms is providing the tools necessary to precisely measure $I B D_{\mathrm{G}}$ with genetic markers in any organism. We encourage researchers to adopt marker-based measures of $I B D_{\mathrm{G}}$ as large numbers of markers and genome maps quickly become available for non-model organisms.

\section{DATA ARCHIVING}

Our simulation program is available in Dryad: http://dx.doi.org/ 10.5061/dryad.f8p63.

\section{CONFLICT OF INTEREST}

The authors declare no conflict of interest. 


\section{ACKNOWLEDGEMENTS}

MK was supported by the National Science Foundation (NSF) through the Montana Institute on Ecosystems (EPS-1101342) and the Montana Ecology of Infectious Diseases IGERT program (DGE-0504628). GL and FWA were partially supported by NSF grants DEB-0742181 and 1258203. GL was also partially supported by DEB-0723928. We greatly appreciate helpful comments from the reviewers.

Allendorf FW, Hohenlohe PA, Luikart G (2010). Genomics and the future of conservation genetics. Nat Rev Genet 11: 697-709.

Balloux F, Amos W, Coulson T (2004). Does heterozygosity estimate inbreeding in real populations? Mol Ecol 13: 3021-3031.

Broman KW, Weber JL (2000). Characterization of human crossover interference. Am J Hum Genet 66: 1911-1926.

Chapman N, Thompson E (2003). A model for the length of tracts of identity by descent in finite random mating populations. Theor Popul Biol 64: 141-150.

Daetwyler H, Hickey J, Henshall J, Dominik S, Gredler B et al. (2010). Accuracy of estimated genomic breeding values for wool and meat traits in a multi-breed sheep population. Anim Prod Sci 50: 1004-1010.

Darwin C (1868). The Variation of Animals and Plants Under Domestication, Vol 2. Murray: London.

Davey JW, Hohenlohe PA, Etter PD, Boone JQ, Catchen JM, Blaxter ML (2011). Genomewide genetic marker discovery and genotyping using next-generation sequencing. Nat Rev Genet 12: 499-510.

Dumont B, Payseur B (2008). Evolution of the genomic rate of recombination in mammals. Evolution 62: 276-294.

Ellegren $\mathrm{H}$ (2014). Genome sequencing and population genomics in non-model organisms. Trends Ecol Evol 29: 51-63.

Fisher RA (1965). The Theory of Inbreeding, 2nd ed. Oliver \& Boyd: Edinburgh.

Forstmeier W, Schielzeth H, Mueller JC, Ellegren H, Kempenaers B (2012). Heterozygosity-fitness correlations in zebra finches: microsatellite markers can be better than their reputation. Mol Ecol 21: 3237-3249.

Franklin I (1977). The distribution of the proportion of the genome which is homozygous by descent in inbred individuals. Theor Popul Biol 11: 60-80.

Hill WG (1981). Estimation of effective population size from data on linkage disequilibrium. Genet Res 38: 209-216.

Hill WG, Weir BS (2011). Variation in actual relationship as a consequence of Mendelian sampling and linkage. Genet Res 93: 47-64.

Hoffman JI, Simpson F, David P, Rijks JM, Kuiken T, Thorne MA et al. (2014). Highthroughput sequencing reveals inbreeding depression in a natural population. Proc Nat/ Acad Sci USA 111: 3775-3780.

Ives AR, Whitlock MC (2002). Inbreeding and metapopulations. Science 295: 454-455

Kardos M, Allendorf FW, Luikart G (2014). Evaluating the role of inbreeding depression in heterozygosity-fitness correlations: how useful are tests for identity disequilibrium? Mol Ecol Res 14: 519-530.

Kawakami T, Backström N, Burri R, Husby A, Olason P, Rice AM et al. (2014). Estimation of linkage disequilibrium and interspecific gene flow in Ficedula flycatchers by a newly developed 50k single-nucleotide polymorphism array. Mol Ecol Res 14: 1248-1260.

Keller LF (1998). Inbreeding and its fitness effects in an insular population of song sparrows (Melospiza melodia). Evolution 52: 240-250.
Keller LF, Waller DM (2002). Inbreeding effects in wild populations. Trends Ecol Evol 17: 230-241.

Keller MC, Visscher PM, Goddard ME (2011). Quantification of inbreeding due to distant ancestors and its detection using dense single nucleotide polymorphism data. Genetics 189: 237-249.

Kenney J, Allendorf FW, McDougal C, Smith JLD (2014). How much gene flow is needed to avoid inbreeding depression in wild tiger populations? Proc Biol Sci 281: 20133337.

Kirin M, McQuillan R, Franklin CS, Campbell H, McKeigue PM, Wilson JF (2010). Genomic runs of homozygosity record population history and consanguinity. PLOS ONE 5: e13996.

Leutenegger A-L, Prum B, Génin E, Verny C, Lemainque A, Clerget-Darpoux F et al. (2003). Estimation of the inbreeding coefficient through use of genomic data. Am J Hum Genet 73: 516-523.

McQuillan R, Eklund N, Pirastu N, Kuningas M, McEvoy BP, Esko T et al. (2012). Evidence of inbreeding depression on human height. PLoS Genet 8: e1002655.

McQuillan R, Leutenegger AL, Abdel-Rahman R, Franklin CS, Pericic M, Barac-Lauc L et al. (2008). Runs of homozygosity in European populations. Am J Hum Genet 83 359-372.

Miller J, Malenfant R, David P, Davis C, Poissant J, Hogg J et al. (2014). Estimating genome-wide heterozygosity: effects of demographic history and marker type. Heredity 112: 240-247.

O'Grady JJ, Brook BW, Reed DH, Ballou JD, Tonkyn DW, Frankham R (2006). Realistic levels of inbreeding depression strongly affect extinction risk in wild populations. Biol Cons 133: 42-51.

Pemberton J (2004). Measuring inbreeding depression in the wild: the old ways are the best. Trends Ecol Evol 19: 613-615.

Pemberton JM (2008). Wild pedigrees: the way forward. Proc Biol Sci 275: 613-621.

Polašek O, Hayward C, Bellenguez C, Vitart V, Kolčić I, McQuillan R et al. (2010). Comparative assessment of methods for estimating individual genome-wide homozygosity-by-descent from human genomic data. BMC Genom 11: 139.

Pompanon F, Bonin A, Bellemain E, Taberlet P (2005). Genotyping errors: Causes, consequences and solutions. Nat Rev Genet 6: 847-859.

Purcell S, Neale B, Todd-Brown K, Thomas L, Ferreira MA, Bender D et al. (2007). PLINK a tool set for whole-genome association and population-based linkage analyses. $A m \mathrm{~J}$ Hum Genet 81: 559-575.

Core Team R (2013). R: A Language and Environment for Statistical Computing. R Foundation for Statistical Computing: Vienna, Austria. URL http://www.R-project. org/.

Santure AW, Stapley J, Ball AD, Birkhead TR, Burke T, Slate J (2010). On the use of large marker panels to estimate inbreeding and relatedness: empirical and simulation studies of a pedigreed zebra finch population typed at 771 SNPs. Mol Ecol 19: 1439-1451.

Slate J, David P, Dodds KG, Veenvliet BA, Glass BC, Broad TE et al. (2004) Understanding the relationship between the inbreeding coefficient and multilocus heterozygosity: theoretical expectations and empirical data. Heredity 93: 255-265.

Stam $\mathrm{P}$ (1980). The distribution of the fraction of the genome identical by descent in finite random mating populations. Genet Res 35: 131-155.

Szpiech ZA, Xu J, Pemberton TJ, Peng W, Zöllner S, Rosenberg NA et al. (2013). Long runs of homozygosity are enriched for deleterious variation. Am J Hum Genet 93: 90-102.

Wang J (2014). Marker-based estimates of relatedness and inbreeding coefficients: an assessment of current methods. J Evol Biol 27: 518-520.

Wright S (1922). Coefficients of inbreeding and relationship. Am Nat 56: 330-338.

Supplementary Information accompanies this paper on Heredity website (http://www.nature.com/hdy) 